\title{
Die Fabrik im Fokus - Fazit
}

Rauchende Schlote und kastenförmige Fabrikbauten aus Backstein, Glas und Stahl hatten sich bis zum Vorabend des Ersten Weltkriegs zu einem festen Bestandteil von Siedlungen und Städten im Russischen Reich entwickelt. Die Mehrheit der Bevölkerung hatte jedoch nie die Möglichkeit, durch die Werkstore zu treten und hinter die Fabrikfassaden zu blicken. Das neue visuelle Medium der Fotografie ermöglichte dies, indem es Szenen vor der Kameralinse auf Papier bannte und so Einblicke in Fabriken gab. Bereits in den 186oer Jahren erkannten erste Unternehmer das Potential fotografischer Aufnahmen, um die neu entstandenen industriellen Räume einem breiten Publikum zugänglich zu machen, ohne tatsächlich die Werkstore zu öffnen. In Absprache mit ihren Auftraggebern fertigten Lichtbildner inszenierte Aufnahmen an. Das Chaos, der Schmutz und der Lärm der reellen Werkshallen wichen auf den Bildern weitläufigen, licht- und luftdurchfluteten Räumen. Dank des neuen visuellen Mediums konnten die Industriellen ein Idealbild ihrer Unternehmen präsentieren. Die Fotografien zeigten Fabriken als Wunschbilder, als seien alle Forderungen der Industrialisierungskritiker bereits Wirklichkeit geworden. Veröffentlicht werden durften die Aufnahmen nur, wenn die Betriebsleitung sie genehmigt hatte. So kontrollierte diese das visuelle Erscheinungsbild ihrer Firma in der Öffentlichkeit.

Zahlreiche Elemente der Bildsprache der Industriefotografie übernahmen Lichtbildner von visuellen Vorgängern, von Grafiken oder aus der Malerei. Dazu gehört die Zentralperspektive, die es ermöglichte, die neuen Dimensionen der Innenräume in Szene zu setzen. Gemeinsam ist der Fabrikforotgrafie und ihren Vorgängern außerdem, dass wenn Künstler Wekshallen oder Arbeitsabläufe zeigten, diese meist geschönte waren. Insbesondere Lichtbildner ästhetisierten Außenansichten und Fabrikhallen, indem sie ihre Bildausschnitte so wählten, dass Lagerstätten von Produkten oder Maschinenanlagen mit ihren Schwungrädern, Achsen und Treibriemen symmetrische Kompositionen bildeten. Diese teilweise fast grafisch wirkenden Aufnahmen lassen vergessen, dass Fabriken im 19. Jahrhundert Orte der Disziplinierung waren. Die visuelle Ordnung und Ästhetik der Bilder lässt keinen Gedanken darüber aufkommen, was es für den menschlichen Körper bedeutet, sich dem Rhythmus einer Maschine zu unterwerfen, immer in der Gefahr, durch ein Unglück oder langjährige repetitive Arbeit versehrt zu werden. Die Fotografien verschweigen dem Betrachter, was es für die Menschen bedeutet, sich neuen Zeit- und Arbeitsregimen zu 
unterwerfen, deren maschinisierte Abläufe kaum individuelle Gestaltungsmöglichkeiten ließen.

Ebenso können die symmetrischen Aufnahmen, bei denen Fotografen häufig mit Aneinanderreihung ähnlicher Formen und Motiven arbeiteten, als Versuch interpretiert werden, einen auf den ersten Blick chaotischen Ort $\mathrm{zu}$ sortieren. Der Wunsch nach Standardisierung und einer hier visuellen Ordnung ist ein charakteristisches Merkmal des 19. Jahrhunderts. Vergleichbare Versuche zeigen sich sowohl in den Bemühungen um die Einführung einer auf einen Standard zurückzuführenden weltweiten Zeitrechnung als auch in Bestrebungen von Medizinern, Medikamente oder Gesundheitszustände anhand festgelegter Normen vergleichbar zu machen. ${ }^{1}$ Beim Bau der russischen Eisenbahn ließen die Verkehrsbehörden die Bahnhöfe in regelmäßigen Abschnitten und nach festgelegten Prototypen bauen, so dass Eisenbahnstrecken mit ihrem einheitlichen Bild als bauliche Einheit erschienen. ${ }^{2}$ Zwar handelte es sich bei der Herausbildung der visuellen Bildsprache der Industriefotografie nicht um eine gezielt forcierte Entwicklung, bei der die beteiligten Akteure in direktem Austausch miteinander standen. Dennoch spiegeln die ästhetisierten Aufnahmen das Bedürfnis von Fotografen und Unternehmern, die Fabrikräume zumindest auf den einzelnen fotografischen Aufnahmen zu ordnen und in ein einheitliches, harmonsiches Bild zu bringen.

Diese Abbildungsstrategien waren in ganz Westeuropa sowie in den USA verbreitet und können als transnationale Bildsprache der Industrie im 19. Jahrhundert bezeichnet werden. Die Ergebnisse der vorligenden Arbeit verdeutlichen, wie eng vor dem Ersten Weltkrieg die Verflechtungen zwischen dem Zarenreich und den westeuropäischen Ländern waren. Landesspezifische Besonderheiten lassen sich insbesondere auf der Ebene der Motive beobachten. Im russischen Fall gehören hierzu die zahlreichen Fotografien von Kirchen, die auf die wichtige, staatstragende Rolle der russisch-orthodoxen Kirche und Religion verweisen. Eine weitere Besonderheit sind Abbildungen menschenleerer Schlafsäle, die für russische Betrachter die Fürsorge des Arbeitgebers symbolisierten und angesichts der katastrophalen Lebensbedingungen der einfachen russischen Bevölkerung und des großen Mangels an Wohnraum als fortschrittlich erscheinen mussten.

1 Zur Standardisierung der Zeit: Osterhammel: Verwandlung der Welt, S. 118-121. Zur Standardisierung in der Medizin: Katharina Kreuder-Sonnen: Wie man Mikroben auf Reisen schickt. Zirkulierendes bakteriologisches Wissen und die polnische Medizin 1885-1939, Tübingen 2018.

2 Schenk: Russlands Fahrt in die Moderne, S. $125^{-126}$ 
Darüber hinaus sind insbesondere die visuellen Leerstellen bezeichnend. Während sich in Frankreich und Deutschland die Herrschenden gerne im Kontext von Fabriken abbilden ließen, fehlen diese Aufnahmen im Russischen Reich bis 1914. Das ist umso bemerkenswerter, als dass die Zaren durchaus Industriebetriebe besichtigten. Die Skepsis gegenüber der Industrialisierung, die sich in gesellschaftlichen Diskussionen äußerte, dürfte ein Grund für diese visuelle Leerstelle gewesen sein.

Von dieser Leerstelle abgesehen schlug sich die Kritik an der Industrialisierung hingegen nur selten in den Bildwelten des Zarenreichs nieder. Es überwogen positive Darstellungen von Fabriken. Dies galt für den redaktionellen Teil russischer Illustrierter ebenso wie für firmeneigene Publikationen. Zwar erschienen Sensationsmeldungen über Unglücke in Unternehmen, überwiegend über Brände. Aber nur vereinzelt berichteten Journalisten über Betriebe und deren Produktion oder äußerten gar Kritik an den bestehenden Arbeits- und Lebensbedingungen der Belegschaft. Statt der Pressefotografie war in Illustrierten die Karikatur das Medium, um sich mit den Schattenseiten der Industrialisierung auseinanderzusetzen.

Fotografien von Arbeitsunfällen erreichten die Öffentlichkeit kaum, weil Unternehmer kein Interesse daran hatten, diese in Umlauf zu bringen. Zwar existieren Aufnahmen von Nachtasylen oder aus Elendsvierteln, doch die Herausgeber dieser Fotografien stellten keinen expliziten Zusammenhang zur russischen Industrialisierung her. Herausgeber sozialistischer Publikationen waren bis 1914 unter anderem aus Kostengründen nicht in der Lage, ihre Blätter mit gedruckten Fotografien zu illustrieren. Dies führte zu einer Situation, in der einem vielschichtigen textuellen und mündlichen Diskurs über die Schattenseiten der Industrialisierung ein überwiegend positiv gefärbtes visuelles Bild der russischen Industrie gegenüberstand.

Mochte sich die Industrialisierung im Zarenreich später und langsamer entwickelt haben als jenseits der Grenzen des Russischen Reichs, lagen russische Fabrikanten und Großunternehmer bei der Verwendung der neuen Bilder mit den meisten ihrer westeuropäischen und amerikanischen Kollegen gleichauf ein weiteres Zeichen für die engen Verbindungen zwischen Unternehmern im Russischen Reich und ihren europäischen und amerikanischen Kollegen. Industrie- und Weltausstellungen boten beispielsweise die Möglichkeit, sich im direkten Vergleich mit der (inter-)nationalen Konkurrenz einen Eindruck darüber zu verschaffen, welches neue Potential die fotografischen Bilder boten.

Mit der Verbreitung der Fotografie bildeten sich neue Verwendungsweisen und Praktiken für die fotografischen Aufnahmen heraus. Unternehmer ließen mit ihnen Bauarbeiten, Unfälle oder wichtige Ereignisse wie Besuche bedeutender Persönlichkeiten oder Jubiläumsfeierlichkeiten dokumentieren. 
Sie nutzten die Bilder für Reklamepublikationen oder verschenkten Abzüge und Fotoalben an ihre Geschäftspartner, politische Würdenträger oder langjährige Mitarbeiter. Nicht in allen Bereichen ersetzten die neuen Bilder ihre überwiegend grafischen Vorgänger. Gerade in Fachzeitschriften überwogen als Illustrationen für Werbeanzeigen bis ins 20. Jahrhundert hinein Druckgrafiken. Zudem reichte keine Fotografie an die Vielschichtigkeit technischer Zeichnungen heran, die die Funktionsweise einer Anlage sowie Details der Konstruktion auf einem Blatt wiedergeben konnten.

Die große Offenheit, mit der viele Fabrikanten der Fotografie begegneten, und wie schnell sie das Medium für ihre Interessen einsetzten, hatte unterschiedliche Gründe. Zunächst waren Unternehmer es gewohnt, sich mit technischen Veränderungen auseinanderzusetzen. Dies bedingte, dass sie technischen Neuerungen prinzipiell offener gegenüberstanden als industrialisierungskritische Kreise der russischen Gesellschaft. Darüber hinaus waren Fabrikfotografien ideal, um die eigene Fortschrittlichkeit unter Beweis zu stellen: Ihrem Verfahren nach technische Bilder zeigten ihre Motive überwiegend technische Maschinen und Industrieanlagen. Hinzu kam, dass das Image der Fotografie als modernes und mechanisch reproduzierbares Bildmedium bestens geeignet war, um mittels der Form und den Bildinhalten der Industriefotografien die Modernität der Auftrag gebenden Unternehmen zu unterstreichen.

Lose Einzelabzüge, Firmenalben oder mit Fotografien illustrierte Festschriften erreichten in der Regel nur einen begrenzten Teil der russischen Gesellschaft, nämlich überwiegend Personen der Mittel- und Oberschicht, die häufig bereits einen Bezug zur Industrie hatten. Um weitere Kreise der Bevölkerung und damit potentielle Konsumenten auf den eigenen Betrieb und dessen Produkte aufmerksam zu machen, setzten besonders Unternehmer aus der Nahrungs- und Luxuswarenindustrie auf Werbeartikel in populären illustrierten Zeitschriften. Aber auch andere Akteure hatten ihre eigenen Interessen, warum sie Industriefotografien anfertigten und vertriebe und damit das Bild von Fabriken im Zarenreich mitbeeinflussten. Dies galt auch für die Herausgeber illustrierten Postkarten. Auf russischen Landschaftspostkarten entwickelten sich Fabrikansichten zu einem beliebten Motiv. Je nach Region erschienen von Industrieanlagen mehr Ansichtskarten als vom örtlichen Kreml oder von verschiedenen Kirchen. Gerade in der russischen Provinz, abseits der großen urbanen Zentren, waren Korrespondenzkarten mit Fabrikmotiven bei den Käufern beliebt. Lokale Postkartenproduzenten ergriffen die Möglichkeit, ihre Region auf den Karten als industrialisiert und fortschrittlich darzustellen, unabhängig davon, ob anderer Standorte sie möglicherweise bereits mit ihrer Wirtschaftsleistung überflügelt hatten. 
Industrieaufnahmen waren kein Genre, mit dem Lichtbildner innerhalb der fotografischen Zunft Ansehen erlangten. Fabrikfotografien zählten nicht zur Kunstfotografie, sondern waren eine Form der Gebrauchsfotografie. Möglicherweise spiegeln die unterschiedlichen Bereiche und Medien, in denen die Bilder von Fabriken und Industrieanlagen Verwendung fanden, daher umso mehr die großen, gesellschaftlichen Veränderungen, mit denen sich die Bewohner des Zarenreichs im ausgehenden 19. und frühen 20. Jahrhundert konfrontiert sahen.

Die Entwicklung der (Bild-)Postkarte trug der gesteigerten Mobilität Rechnung, die Teil der Industrialisierung war. Je weiter entfernt voneinander Familienmitglieder lebten, desto mehr waren sie auf postalische Kommunikationsmittel angewiesen, um miteinander in Kontakt zu bleiben. Dies ermöglichten die preiswerten Korrespondenzkarten und führten darüber hinaus auf der Bildebene zu einer interregionalen Verflechtung innerhalb des Zarenreichs. Aufnahmen entlegener Regionen reisten durch das Imperium und wurden dadurch zumindest scheinbar zugänglich. Landschaftspostkarten mit gedruckten Fotografien regionaler Sehenswürdigkeiten und Stadtansichten zeugen gleichzeitig vom erwachenden Regionalbewusstsein. Teil dieses regionalen Selbstverständnisses, insbesondere in der Provinz, waren häufig auch Fabriken und Industrieanalgen. Der Ausbau des Eisenbahn- und Telegrafennetzes veränderte Raum- und Zeitwahrnehmung und verstärkte das Gefühl, in einer Periode immer schneller eintretender Veränderungen zu leben. Die ständige Unsicherheit führte zu einem großen gesellschaftlichen Interesse für Geschichte, die Verlässlichkeit suggerierte. Diese Sehnsucht nach Kontinuität und Beständigkeit schlug sich in aufwendigen Jubiläums- und Firmenjubiläumsfeiern sowie in den Firmenfestschriften nieder, die zu diesen Anlässen erschienen. Die Autoren stellten die Firmengeschichten und langjährige Tradition möglichst eindrücklich dar und ließen die Betriebe gegenüber ihren Geschäftspartnern als verlässlich und vertrauenswürdig erscheinen.

Gleichzeitig spiegelten die Festlichkeiten an Firmenjubiläen die stark hierarchischen und paternalistischen Strukturen, die in der Gesellschaft des Zarenreichs verbreitet waren. In den meisten Fällen feierten Firmenleitung, Meister und Arbeiter getrennt voneinander und erhielten unterschiedliche Präsente zur Erinnerung an das Ereignis. Vor dem Hintergrund einer erstarkenden Arbeiterbewegung und vermehrt auftretender Streiks erkannten die Unternehmer Jubiläen jedoch auch als Chance, um ein Gemeinschaftsgefühl und Loyalitäten gegenüber dem Betrieb zu wecken. Jubiläen und die in diesem Kontext entstandenen Fotografien und illustrierten Festschriften hatten das Potential, drohenden sozialen Unruhen vorzubeugen. 
Die Entwicklung der illustrierten Presse und die Ausbildung eines professionalisierten Reklamewesens, an dem sich auch Fabrikanten maßgeblich beteiligten, zeugen von der zunehmenden Bedeutung des Konsums für das Zarenreich. Die illustrierten Zeitschriften waren Produkte des Massenkonsums. Gleichzeitig waren ihre Werbeteile Plattformen, auf denen Industrielle ihre Produkte und Firmen als Marken zu platzieren suchten.

Die Fotografien selbst dokumentierten den rasanten technischen Fortschritt im Zarenreich. Beschränkten sich die ersten Fabrikaufnahmen der 1860er Jahre auf Außenansichten, waren Lichtbildner zehn Jahre später bereits in der Lage, Innenaufnahmen anzufertigen. An die Stelle einzelner, großer Dampfmaschinen traten Fabrikhallen voller Maschinenanlagen. Der Mensch verlor angesichts dieser Übermacht der Technik immer mehr an Bedeutung, außer er musste im Kontext der Arbeiterfürsorge das Engagement der Fabrikleitung belegen oder mit Gruppenaufnahmen die Größe der Belegschaft und Prosperität eines Betriebs nachweisen.

Nicht zuletzt zeugen die Aufnahmen von der Etablierung des kapitalistischen Wirtschaftsmodells im Russischen Reich. Die Existenz zahlreicher aufwendiger Firmenalben belegen die Herausbildung von Großunternehmen. Nur erfolgreiche Fabriken mit hohen Gewinnen konnten sich das kostspielige Engagement eines Lichtbildners und die Herstellung von Abzügen und kostbar ausgestatteten Alben leisten.

Der Erste Weltkrieg stellte für die Bedeutung der Fabrikfotografie einen Wendepunkt dar. Bis ins Jahr 1914 wurde die Verwendung von Industriefotografien in erster Linie durch ökonomische Aspekte bestimmt: Firmen nutzten Fotografien zur Selbstpräsentation und zu Werbezwecken, Zeitschriften versuchten mit reißerischen Aufnahmen und Berichten über Brandkatastrophen Leser zu finden und Postkartenherausgeber wollten ihre Karten an die lokale Bevölkerung verkaufen. Außerdem hatten Fotografen ein Interesse daran, lukrative Aufträge von Firmen zu erhalten, und wandten sich darum teilweise selbst mit Vorschlägen an Firmenleitungen. Politische Akteure beteiligten sich kaum an der Herstellung der industriellen Bildwelten des Zarenreichs.

Nach Beginn des Ersten Weltkriegs änderte sich die Funktionsweise von Industriefotografien. In russischen Zeitschriften traten Fabrikfotografien nun im offiziellen Redaktionsteil in Erscheinung, flankiert von patriotischen Berichten. Zar Nikolaus II., der die Industrie bislang für seine Selbstpräsentation nicht eingesetzt hatte, erkannte deren kriegswichtige Bedeutung und zeigte sich mit Arbeitern und in Fabrikhallen. Politisches Kalkül und propagandistische Interessen verhalfen Fabrikfotografien zu einem neuen Stellenwert. Dank der militärischen Verbündeten und über internationale Netzwerke von Bildagenturen erreichten das russische Publikum Fabrikfotografien 
aus dem Ausland. Sie zeigten eine neue Bildsprache, denn die englischen und französischen Lichtbildner rückten mehr und mehr Arbeiter und besonders Arbeiterinnen ins Zentrum der Aufmerksamkeit und ihrer Aufnahmen.

Der propagandistische Einfluss auf die Produktion von Industriefotografien nahm weiter zu, nachdem sich die Bolschewiki in der Oktoberrevolution 1917 an die Spitze des Staats geputscht hatten. Ebenso intensivierte sich die Bedeutung, die Arbeiterinnen und Arbeiter auf Fabrikfotografien einnahmen, nachdem die Bolschewiki sie zur neuen gesellschaftlichen Elite erklärt hatten. Und doch bedeuteten die politische Zäsur und die neue ideologische Rahmung der Industriefotografie nicht auf allen Ebenen einen radikalen Bruch mit der Entwicklung des Genres im Zarenreich. Zahlreiche Akteure und vorrevolutionäre Bildtraditionen prägten die russische Fabrikfotografie auch nach 1917. Zwar veränderte sich nach der Oktoberrevolution die Bildsprache mit neuen Frosch- und Vogelperspektiven sowie Inszenierungsstrategien und die Bandbreite der Motive nahm zu, dennoch blieben die Kernaussagen der Bilder bestehen. Sie strahlten weiterhin Begeisterung für den technischen Fortschritt aus. Sowjetische Illustrierte stellten keine Plattformen für die Reklame einzelner Fabrikanten mehr zur Verfügung. Sie propagierten stattdessen die staatlichen Bemühungen um eine forcierte Industrialisierung und warben um Sympathien für den neuen sowjetischen Staat und sein Wirtschaftssystem.

Nichtsdestotrotz blieb in den Fabrikfotografien das Bestreben, maschinelle und industrielle Stärke zu demonstrieren, erhalten. Die sauberen Arbeiter der Vorkriegszeit mit ihren weißen Hemden wichen heroischen Stoßarbeitern, die schweiß̈̈berströmt und schwarz von Kohlestaub waren. Die vormals fabrikeigene Zensur wich einer staatlichen Zensur der Bolschewiki. Sowjetische Lichtbildner blendeten in ihren Bildreportagen weiterhin Monotonie und Unfallgefahr aus, die von den industriellen Anlagen ausging, weil die forcierte Industrialisierung nach der Oktoberrevolution zum Erfolg verpflichtet war. An die Stelle der ernsten Gesichter der Belegschaft trat bereits mit dem Ersten Weltkrieg das Lächeln der Arbeiterinnen und Arbeiter, die begeistert an der Heimatfront beziehungsweise für den neuen Staat tätig waren. Die russische Fabrikfotografie bewahrte sich auch unter den neuen Machthabern ihr positives Narrativ und den Glauben an Technik und Maschinen. 